\title{
ORIGINAL ARTICLE \\ Spinal cord and spine trauma in a large teaching hospital in Ghana
}

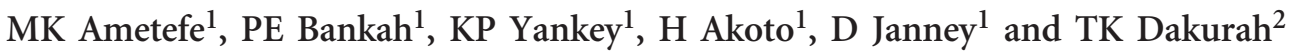

Introduction: Spinal cord injury (SCl) is a devastating injury, with its effect going beyond the injured patient to the care givers and family and with economic implications that can be long lasting. The study determined the occurrence and patterns of SCl and spine injury patterns, treatment and treatment outcomes in a large tertiary health facility in Ghana.

Methods: This was a retrospective review of health records of patients at the Korle bu Teaching Hospital, Ghana. Data on 185 patients were collected over a period of 18 months from September 2012 to February 2014. Data were collected on basic demographic characteristics (age distribution), cause of SCI, type and severity of injuries, mode of transportation to the hospital and treatment modalities. In addition, data were collected on the imaging techniques used, waiting time and delays encountered, complications and follow-up. Descriptive statistics were used to analyze data using Windows Excel 2007 version.

Results: A total of 185 patients were treated in the study period, 125 (67.6\%) patients had cervical spine injury, 33 (17.8\%) had thoracic spine injuries and 27 (14.6\%) had lumbar injuries. In all, 141 (76\%) were males. The age range of patients was 4 years to 86 years; mean age was $36.25 \pm 13.62$ years. Spinal injuries were most common in the 31-45-year age group, followed by 16-30-year group. Most prevalent cause of spinal injury was road traffic accident (RTA), 130 (70.3\%), whereas assault was the least common, $5(2.7 \%)$. Delay in getting imaging studies conducted was high; 43 (23\%) of the computed tomography scans required were performed after $48 \mathrm{~h}$ of admission. Only 76 (41\%) patients were able to afford the cost of magnetic resonance imaging. Pressure sore (23\%) and pneumonia (21\%) were the most common complication during admission.

Conclusion: RTA was the most common cause of spinal injuries and occurred in the relatively young population, especially among men. Structured public education and enforcement of road safety measures are imperative. Rapid response to management of patients with $\mathrm{SCl}$ at the teaching hospital needs attention by hospital management.

Spinal Cord (2016) 54, 1164-1168; doi:10.1038/sc.2016.57; published online 3 May 2016

\section{INTRODUCTION}

Spinal cord injury (SCI) is a devastating injury, with its effect going beyond the injured patient to the care givers and family and with economic implications that can be long lasting. ${ }^{1-7}$ The disability that it brings has been known to have long lasting effects with very little available to restore its functions ${ }^{7}$. Beyond the temporary or permanent loss of motor, sensory and autonomic functions, the patient may suffer psychologically, and the injury may demand difficult psychological and social adjustments from family members as well. ${ }^{8}$

Globally, the epidemiology of SCI has been documented from various countries and subregions. However, very little is documented about the characteristics of SCI and spine trauma in Ghana and their treatment outcomes. This justifies our research into SCI in our hospital. Korle bu Teaching Hospital is an appropriate center, as it is one of the few hospitals that manage SCI in the southern part of the Country.

The study was aimed at evaluating the SCI and associated spine injury patterns, demographic variables, treatment offered and their outcomes. It also looked at the imaging studies used in the management of patients.

\section{MATERIALS AND METHODS}

The study was conducted over 18 months from September 2012 to February 2014 in the Neurosurgical Unit of the Korle bu Teaching Hospital, Accra, Ghana.

People for this analysis were patients who were injured in the period of study and managed at the teaching hospital. It excluded all patients with deficits attributable to pathological fractures or tumors. Data extracted included the age, gender, spinal cord level of injury, American Spinal Injury Association Impairment scale (AIS) on admission and imaging studies performed during the period of treatment. Data were also collected on mode of transport into the facility, cause of injury, type of treatment employed, the complications encountered during admission period and the neurological changes that were observed in managing the patients.

Data collected were analyzed with simple descriptive statistics (frequencies and proportion) and presented as Tables. Data were analyzed with Microsoft Excel, 2007.

Surgical intervention was offered to all patients with instability and/or compression of cord and nerve roots at point of injury. Anterior cervical discectomy and fusion with iliac crest bone graft with instrumentation in most cases and where applicable, a corpectomy was employed. Some patients had posterior instrumentation with lateral mass screws. Thoracic and lumbar spine injuries were instrumented from a posterior approach in all cases. A posterior spinal fusion was performed and sometimes pedicle subtraction osteotomy,

${ }^{1}$ Neurosurgery Unit, Department of Surgery, Korle bu Teaching Hospital, Accra, Ghana and ${ }^{2}$ Department of Surgery, School of Medicine and Dentistry, University of Ghana, Legon, Ghana

Correspondence: Dr M Ametefe, Neurosurgery Unit, Department of Surgery, Korle bu Teaching Hospital, PO Box KB77, Korle bu, Accra, Ghana.

E-mail: ametmk@yahoo.co.uk

Received 9 September 2015; revised 24 January 2016; accepted 28 March 2016; published online 3 May 2016 
facetectomy and anterior fusion with hams cage were used where it was applicable. All hams cage was put in place from a posterior approach.

\section{RESULTS}

A total of 185 patients were managed in the period of study, giving an average of 10.3 per month. Of these, $141(76.2 \%)$ were males, giving a male-to-female ratio of 3.2:1. Ages ranged from 4 to 86 years, with the age group most affected being 31-45 years (44\%) followed closely by 16-30-year group (31\%). These two groups form $75 \%$ of the SCIs managed.

The age distribution with the various injuries is shown in Table 1.

Table 2 shows that a total of 125 patients had cervical spine injury, forming 67.5\%, whereas thoracic and lumbar spine injuries had 33 and 27 patients forming $18.0 \%$ and $14.5 \%$, respectively. Of the cervical spine injuries, $53(42 \%)$ were complete, 40 (32\%) were incomplete and $32(26 \%)$ were neurologically intact. There were $7(3.7 \%)$ odontoid fractures, $6(3.2 \%)$ hangman's fractures and $11(6 \%)$ locked facets seen in the period of study.

Total thoracic spine injury seen in the period was 33 . This included the thoracolumbar junction injuries, which were 8 in number. Seventeen $(52 \%)$ were complete injuries, whereas incomplete and intact were $9(27 \%)$ and 7 (21\%), respectively. Lumbar region injuries were 27 , of which $4(15 \%)$ were complete injuries and incomplete and intact injuries were 13 (48\%) and 10 (37\%), respectively.

Road traffic accidents (RTAs; 129 (69.7\%)) and falls (45, 24.3\%) were the most common causes of trauma (Table 2). In sub-classifying the RTA, 98 (76\%) were passengers or drivers in vehicles, $19(14.7 \%)$

Table 1 Age distribution of patients

\begin{tabular}{lcccr}
\hline Age, years & C-Spine, n (\%) & T-Spine, n (\%) & L-Spine, n (\%) & Total, n (\%) \\
\hline $0-15$ & $4(2.2)$ & $0(0)$ & $2(1.1)$ & $6(3.2)$ \\
$16-30$ & $35(18.9)$ & $13(7)$ & $9(4.9)$ & $57(30.8)$ \\
$31-45$ & $57(30.8)$ & $16(8.6)$ & $8(4.3)$ & $81(43.8)$ \\
$46-60$ & $23(12.4)$ & $3(1.6)$ & $6(3)$ & $32(17.3)$ \\
$61-75$ & $5(2.7)$ & $1(0.5)$ & $1(0.5)$ & $7(3.8)$ \\
$>75$ & $1(0.5)$ & $0(0)$ & $1(0.5)$ & $2(1.1)$ \\
Total & $125(67.6)$ & $33(17.8)$ & $27(14.6)$ & $185(100)$ \\
\hline
\end{tabular}

Abbreviations: C-Spine, cervical spine; L-Spine, lumbar spine; T-Spine, thoracic spine.

Table 2 Causes of spinal injuries in patients, Korle-Bu Teaching Hospital

\begin{tabular}{lcc}
\hline Causes & Number & Percentage (\%) \\
\hline RTA & 129 & 69.70 \\
Falls & 45 & 24.30 \\
Industrial accidents & 6 & 3.20 \\
Assaults & 5 & 2.70 \\
Sports and leisure & 0 & 0.00 \\
Gunshot injury & 0 & 0 \\
Total & 185 & \\
& & \\
Distribution of causes of injury in RTA & 98 & 76 \\
MVA (passengers and drivers) & 19 & 14.70 \\
Motorbikes & 10 & 7.80 \\
Pedestrian & 2 & 1.60 \\
Bicycle & 129 & 100 \\
Total &
\end{tabular}

Abbreviation: RTA, road traffic accident. were riders or pillion riders on motorbikes, 10 (7.8\%) were pedestrians and $2(1.6 \%)$ were bicycle riders. There were no injuries recorded from gunshot injuries in the period studied. There were $6(3.2 \%)$ industrial-related injuries and $5(2.7 \%)$ assault cases.

AIS grade A was the most frequent and most severe injury category seen in $74(40 \%)$ cases as in Table 3. Grade B injuries were seen in $13(7.0 \%)$, grade $\mathrm{C}$ in $16(8.6 \%)$ and grades $\mathrm{D}$ and $\mathrm{E}$ seen in $33(17.8 \%)$ and $49(26.5 \%)$, respectively. Fifty-three $(71.6 \%)$ of the AIS A had cervical cord injuries.

As shown in Table 4, a total of 33 patients (17.84\%) had associated injuries.

Mode of transportation of patients to the hospital was mainly by ambulance service in $105(56.8 \%)$. This was followed by private cars, $47(25.4 \%)$ and taxis, $16(8.7 \%)$. Five patients were transported with both airplane and ambulance and therefore captured under airplane as their mode of transport. Airplane transport was either by the military aircraft or the commercial domestic flight in two cases. Twelve patients had no record of their mode of transport. Ninety-three of the patients were referred and transferred from other health facilities. Only $7 \%$ came directly to the hospital from injury point.

Imaging studies requested to help evaluate patients were $\mathrm{X}$-rays, computed tomography (CT) scans and magnetic resonance imaging (MRI). The study assessed the time period between admission and acquisition of images (Table 5). Almost all patients eventually had X-rays performed; however, $137(74.1 \%)$ patients had X-rays performed after 2 weeks of admission. More significantly, only 109 (58.9\%) patients had CT scans, and 76 (41.1\%) had MRI scans performed in the same 2-week period. Again after $24 \mathrm{~h}$ of admission, $113(61.1 \%)$ cases had X-rays, and only $21(11.4 \%)$ had CT scans performed.

In the management of the cases seen, 87 (47\%) had surgical intervention and 98 (53\%) cases were managed non-operatively as in Table 6.

The most common complication seen while managing the patients on admission was pressure sores seen in $43(23 \%)$ of patients, which was common in those with complete cervical spine injury, 27 (63\%). Pneumonia was the second most common complication, 39 (21\%),

Table 3 ASIA impairment scale

\begin{tabular}{lc}
\hline Scale category & Number (\%) \\
\hline A & $74(40.0)$ \\
B & $13(7.0)$ \\
C & $16(8.6)$ \\
D & $33(17.8)$ \\
E & $49(26.5)$ \\
Total & $185(100)$ \\
\hline
\end{tabular}

Abbreviation: ASIA, American Spinal Injury Association.

Table 4 Associated injuries in patients

\begin{tabular}{lc}
\hline Type of injury & Number (\%) \\
\hline Head injury & $13(7.0)$ \\
Laceration/avulsions & $11(5.9)$ \\
Limb and facial bone fractures & $8(4.3)$ \\
Chest injuries & $9(4.9)$ \\
Blunt abdominal injury & $2(1.1)$ \\
Burns & $1(0.5)$ \\
\hline
\end{tabular}


Table 5 Time period between admission and conduct of imaging test

\begin{tabular}{lrrr}
\hline $\begin{array}{l}\text { Time period between admission and conduct of } \\
\text { Imaging test }\end{array}$ & X-rays & CT scan & MRI scan \\
\hline$<2 \mathrm{~h}$ & & & \\
$2-6 \mathrm{~h}$ & 29 & 15 & 22 \\
$6.1-12.0 \mathrm{~h}$ & 20 & 0 & 4 \\
$12.1-24.0 \mathrm{~h}$ & 20 & 5 & 1 \\
$24.1-48.0 \mathrm{~h}$ & 12 & 22 & 17 \\
$>48$ h-1 week & 8 & 53 & 17 \\
$>1-2$ weeks & 4 & 13 & 5 \\
Total & 137 & 109 & 76 \\
\hline
\end{tabular}

Abbreviations: CT, computed tomography; MRI, magnetic resonance image.

Table 6 Intervention applied in spinal injury patients in the hospital

\begin{tabular}{lccc}
\hline \multirow{2}{*}{ Region of injury } & Surgical intervention & Non-operative management & \multirow{2}{*}{ Total } \\
\cline { 2 - 3 } & Number (\%) & Number (\%) & \\
\hline Cervical spine & $48(38.4)$ & $77(61.6)$ & $125(67.7)$ \\
Thoracic spine & $17(51.5)$ & $16(48.5)$ & $33(17.8)$ \\
Lumbar spine & $22(81.5)$ & $5(18.5)$ & $27(14.6)$ \\
Total & $87(47.0)$ & $98(53.0)$ & $185(100)$ \\
\hline
\end{tabular}

Table 7 Complications seen in patients with spinal injuries at the Korle-Bu Teaching Hospital

\begin{tabular}{lc}
\hline Complication & Number (\%) \\
\hline Pneumonia & $39(21.1)$ \\
DVT & $9(4.9)$ \\
PE & $2(1.1)$ \\
UTI & $34(18.4)$ \\
Pressure sores & $43(23.2)$ \\
Upper Gl bleed & $2(1.1)$ \\
Asthma & $2(1.1)$ \\
Heamaturia & $11(5.9)$ \\
\hline
\end{tabular}

Abbreviations: DVT, deep vein thrombosis; GI, gastrointestinal; PE, pulmonary embolism; UTI, urinary tract infection.

followed by urinary tract infection, 34 (18\%). Other complications are shown in Table 7. In all, 29 (16\%) patients died during the admission period.

Follow-up visits after discharge were very poor. In the 6 months after discharge, only $53(29 \%)$ patients came for any follow up. Of these, $28(53 \%)$ reported in the first 4 weeks, 12 (23\%) in 3 months, $6(11 \%)$ in 6 months and $7(13 \%)$ in 1 year. Only 13 patients made more than one follow-up visit to the hospital after discharge. Even though other complications such as persistent spasms, contractures, stuck urethral catheters and severe anemia were recorded, postdischarge complications could not be meaningfully assessed because of failure to get patients for follow-up visits.

No improvement was recorded for the complete spine-injured patients who came for follow-up. Thirteen out of the fifteen incomplete cervical spine-injured patients who came for follow-up had improved and were walking with aid with some spastic gait within 6 months of review. Out of the six incomplete thoracic spine injury patients reviewed, three were walking independently and three with aid, and of the four lumbar spine patients who turned up for review, three were walking independently and one with a limp.

\section{DISCUSSION}

SCI is one of the major injuries managed by the Neurosurgical team in Korle bu Teaching Hospital. In 18 months, the unit managed more than 185 cases of spine injuries with an average of 10.3 cases every month. It has been difficult to establish whether there has been a rise in the number of cases or not, as there has been no found literature to compare with. Anecdotal observation suggests that there has been a progressive increase in the numbers being managed annually.

Knowledge of incidence and prevalence of SCI is important both because of their high personal, bio-psychological impact and because of their high socio-economic consequences, short term as well as long term. ${ }^{9}$ Important as they may be, it is impossible to establish them from our findings. Not all SCIs from the peripheral hospitals receive treatment in a tertiary institution. A number of them end up with the traditional healers and bone setters. In addition to this, some patients also request discharge home against medical advice even before images can be done to establish the full extent of the injury. A further study with a complete survey of spine injuries over the entire country may give a better picture. To the best of our knowledge, this is the first study that is looking at SCI in Ghana.

As noted in other publications, there was a male preponderance. ${ }^{1,4,6,10,11}$ The number of males with SCI in the period was more than three times that of the females. The male, being more risk taking, is prone to injury in general compared with the females. On the contrary, a study of spine trauma in Australia had the proportion of male to female to be $68.7 \%$ and $31.3 \%$, respectively. ${ }^{12}$

There was a predominance of cervical cord injuries (67.5\%), which corresponds to data from other countries. ${ }^{2,4,7,11}$

Our study showed more young active age group being affected by SCI. Seventy-five percent of the injuries were between the ages of 16 and 45 years. This conforms to the pattern depicted in other studies, ${ }^{3,4,7,13}$ and it emphasizes the severity of loss of manpower, productivity due to invalidation and sometimes death to the family and the economy of the nation.

Road traffic injuries remained the primary cause on SCIs in our environment, accounting for $69.7 \%$ of cases. Although this is true for most other publications in the subregion, our findings are the highest noted so far. ${ }^{1,2,4,7}$ The main known and documented causes of RTAs in the subregion include bad maintenance of roads, unsafe driving, poor enforcement of traffic rules and road unworthy vehicles. ${ }^{7}$ Thus, reducing RTA alone in Ghana is likely to subsequently reflect in a reduction in SCIs significantly.

It is difficult to explain the absence of injuries from gunshot injuries in our study, as violence with guns is thought to be on the rise. In South Africa, as reported by Velmahos et al., penetrating spinal injuries accounted for $66.5 \%$ of all spinal injuries, and gunshots account for about $13 \%$ of their spine injuries. ${ }^{14}$ Our conclusion is that gun use in Ghana is not as prevalent as it was thought to be. Contrary to the findings of Cripps et al., violence/self-harm was not significant in our work. ${ }^{15}$ Notable is the industrial injuries attributable to poor and unsafe work environments with non-adherence to any safety precautions and checks that makes most industrial workers prone to injuries.

We expected $93 \%$ of our patients to have been transported in an ambulance, as they were referred from other health facilities. On the contrary, only $56.8 \%$ had the service of an ambulance. Our studies revealed that patients were not guaranteed a good mode of transport just because they were first managed in a health facility. Taxis, private 
cars and even motorbikes remain a means of transportation in our health system even for some referred cases. Obviously, optimal care of the spine fractures and their stabilization while being transported in these unconventional means of transport is not possible. It is possible that some patient may develop worsening SCI during transportation. Of a greater concern is the fact that, of the $7.0 \%$ of the patients who came directly from the injury site, none of them had been transported by ambulance service providers. This only implies that the ambulance services are not effective where they are needed most.

\section{Management}

Early stabilization and mobilization with active physiotherapy has been our treatment plan; however, a number of factors remain stumbling blocks in achieving them. Most important is the delay in getting imaging carried out. Reasons for the delays included finance, poor understanding by family that these were essential imaging for planning of any intervention and a socio-economic cultural practice that suggests that the person or body/group to blame for the injury must pay for the treatment. Worse still, our juvenile health insurance does not cover CT scan, MRI images or funding for spine surgeries, making it absolute that funding must be from elsewhere if imaging and surgery are required. As alluded to by Nwankwo et al., adding the cost of implants and surgery to the treatment becomes a financial burden on our patients, and this accounts for our being able to operate only $47.0 \%$ of our patients. ${ }^{4}$ A significant number of patients managed conservatively or discharged against medical advice would have benefited from surgical intervention. As practiced by Andrews et al., single-level non-instrumented fusion was sometimes employed for cervical spine injuries, with good results in patients who could not afford the implants. ${ }^{16}$

\section{Pressure sores}

In SCI, patients have a high risk of developing pressure ulcers because they are neurologically impaired, mostly immobilized and tend to have long hospital stay. ${ }^{11,17}$ Pressure ulcers continue to be our most observed complication of SCI. Sixty-three percent of the patients with pressure ulcers of varying degrees had complete cervical SCI. Two hourly turning of patients with SCI in bed is extremely difficult to achieve in our environment. This is due to poor nurse-to-patient ratio, which can be as poor as two nurses to thirty-two patients at night. These few nurses are overwhelmed with work making basic skin care, good laying of beds and cleaning of the wounds less optimal. In addition to this, pressure dispersing materials/equipments are severely lacking, negating every effort to minimize the pressure ulcers when they have been identified. Although this study did not focus on the detailed reasons for the high rate pressure ulcers in our patients, we tend to agree with Zakrasek et al. who attributed malnutrition, low education and poverty as part of the risk factors predisposing patients to it. ${ }^{17}$ Even after discharge, the complications of their neurological deficits continue to be a major source of concern for us with urinary tract infection, encrustation and blockage of indwelling catheter, pneumonia, anemia and sepsis being some of the causes of readmission into an emergency facility. Getting cultures for treatment can also be a great task in our environment, making culture-sensitive treatment of infections difficult. It is therefore difficult to treat with evidence as suggested by Stickler et al. ${ }^{18}$

As noted in our findings, some of our patients developed deep ${ }^{19}$ vein thrombosis (DVT) and pulmonary embolism in spite of our protocol for anticoagulation of all SCI patients. The diagnosis of DVT followed a clinical evidence of leg swelling. As it is known that SCI patients can develop DVT and pulmonary embolism without any limb swelling, ${ }^{17}$ our numbers may not be a true reflection of the incidence of DVT and pulmonary embolism among hospitalized SCI patients.

More often, the caretakers of the patient also take up the role of being the bread winners. More attention to the patient (with hospital visits) directly implies decreased productivity and income generation for some families. Thus, bread winners of these families may kill the hospital visit for the economic gain. Hospital visits also have an additional cost from transportation of the patient to and from the hospital, paying for investigations and procuring medications after being reviewed. The current health insurance system does not cover a large portion of the needs of the SCI patient.

Another contributory factor for the poor follow-up is patient's unwillingness to visit the hospital even with a very supportive family. This is not all too surprising. A significant number of SCI patients are noted to have long-term psychosocial effects of their injury, and they develop uncooperative behaviors with refusal to accept medication, hospital visits and care. ${ }^{8}$ In the end, more complications develop and sometimes convert a stable SCI patient into a very ill or sometimes moribund patient who needs urgent care and attention. As recommended by Welk et al., we refer our patients for urologic care; however, there is a poor turn up for review with the urologist as well. ${ }^{20}$

Some of our patients are transported into the villages where access to specialized care and physiotherapy is impossible or worse still neglected by the family.

\section{CONCLUSION}

SCI is a devastating injury that affects mainly the young active productive population in our environment. With RTA being the major cause of this injury, efforts must be made to reduce it by directly reducing RTAs. Imaging for the management of patients is a challenge.

We need a detailed data gathering, possibly multi-centered, aimed at establishing a good incidence and prevalent rate.

\section{DATA ARCHIVING}

There were no data to deposit.

\section{CONFLICT OF INTEREST}

The authors declare no conflict of interest.

\section{ACKNOWLEDGEMENTS}

There was no funding/grant for this work and no financial benefit to the authors

1 Frielingsdorf K, Dunn RN. Cervical spine injury outcome - a review of 101 cases treated in a tertiary referral unit. SAMJ 2007; 97: 203-2207.

2 Draulans N, Kiekens C, Roels E, Peers K. Etiology of spinal cord injuries in SubSaharan Africa. Spinal Cord 2011; 49: 1148-1154.

3 Lee BB, Cripps RA, Fitzharris M, Wing PC. The global map for traumatic spinal cord injury epidermiology: update 2011, global incidence rate. Spinal Cord 2014; 52 : 110-116.

4 Nwankwo OE, Uche EO. Epidemiological and treatment profiles of spinal cord injury in southeast Nigeria. Spinal Cord 2013; 51: 448-452.

5 Kawu AA, Olawepo A, Salami A00, Kuranga SA, AbdulHameed S, Esenwah VC. A cost analysis of conservative management of spinal cord-injured patients in Nigeria. Spinal Cord 2011; 49: 1134-1137.

6 Geyh S, Ballert C, Sinnott A, Charlifue S, Catz A, D'Andrea JM et al. Quality of life after spinal cord injury: a comparison across six countries. Spinal Cord 2013. 51: 322-326.

7 Obalum DC, Giwa SO, Adekoya-Cole TO, Enweluzo GO. Profile of spinal injuries in Lagos, Nigeria. Spinal Cord 2009; 47: 134-137.

8 North NT. The psychological effects of spinal cord injury: a review. Spinal Cord 1999; 37: 671-679.

9 Wyndaele M, Wyndaele J-J. Incidence, prevalence and epidemiology of spinal cord injury: what learns a worldwide literature survey? Spinal Cord 2006; 44: 523-529. 
10 Güzelküçük Ü, Kesikburun S, Demir Y, Aras B, Özyörük E, Yılmaz B et al. Demographic and clinical characteristics of patients with traumatic cervical spinal cord injury: a Turkish hospital-based study. Spinal Cord 2014, 1-5.

11 Lyun AO, Malomo AO, Oluwatosin OM, Ademola SA, Shokunbi MT. Pattern of presentation of pressure ulcers in traumatic spinal cord injured patients in University College Hospital, Ibadan. Int wound J 2011; 9: 206-213.

12 Tee JW, Chan CHP, Fitzgerald MCB, Liew SM, Rosenfeld JV. Epidemiological trends of spine trauma: An Australian Level 1 Trauma Centre Study. Global Spine J 2013; 3: 75-84.

13 Ackery A, Tator C, Krassiokov A. A global perspective on spinal cord injury epidemiology. J Neurotrauma 2004; 10: 1355-1370.

14 Velmahos GC, Degiannis E, Hart K, Souter I, Saadia R. Changing profiles in spinal cord injuries and risk factors influencing recovery after penetrating injuries. J Trauma 1995; 38: 334-337.
15 Cripps RA, Lee BB, Wing P, Weerts E, Mackay J, Brown D. A global map for traumatic spinal cord injury epidemiology: towards a living data repository for injury prevention. Spinal Cord 2011; 49: 493-501.

16 Andrews NB, Lawson HL, Odjidja TL. Elective non-instrumented anterior cervical diskectomy and fusion in Ghana: a preliminary report. West Afr J Med 2003; 22: $128-132$.

17 Zakrasek EC, Creasey G, Crew JD. Pressure ulcers in people with spinal cord injury in developing nations. Spinal Cord 2015; 53: 7-13.

18 Stickler DJ, Freneley RCL. The encrusted and blockage of long-term indwelling bladder catheters: a way forward in prevention and control. Spinal cord 2010; 48: 784-790.

19 Waring WP, Karunas RS. Acute spinal cord injuries and the incidence of clinically ocurring thromboembolic disease. Paraplegia 1991; 29: 8-16.

20 Welk B, Chi Tran K, Liu K, Shariff S. The pattern of urologic care among traumatic spinal cord injured patients. Can Urol Assoc J 2014; 8: e805-e809. http://dx.doi.org/10.5489/cuaj.2403. 